\title{
The Application of Aqueduct-100 for Controllable Cervical Dilation
}

\author{
Vanessa Martinellii ${ }^{1}$, Farr Nezhat ${ }^{1,2}$, Michael White ${ }^{1 *}$, Pengfei Wang ${ }^{1}$, Amnon Weichselbaum ${ }^{3}$ and $_{\text {Michael Stark }}^{4}$ \\ ${ }^{1}$ NYU Winthrop Hospital, Mineola, USA
}

${ }^{2}$ Weill Cornell Medical College of Cornell University, USA

${ }^{3}$ NGT Technological incubator, Israel

${ }^{4}$ New European Surgical Academy, Germany

Submission: April24, 2020 ; Published: May 12, 2020

*Corresponding author: Michael White, NYU Winthrop Hospital, Mineola, New York, USA

Abstract

Objective: To evaluate the efficacy of the Aqueduct-100 to dilate the cervix to a predetermined diameter.

Methods: Both mechanical and osmotic methods of cervical dilation pose risks of cervical laceration, creation of a false passage, and uterine perforation. The Aqueduct- 100 is a modified cervical dilator that has a triple balloon catheter with a total diameter of $2.3 \mathrm{~mm}$. This dilator is then inserted through the external and internal cervical os and then secured in place. Dilation balloons were then sequentially inflated until desired diameter. The study included 19 patients undergoing any diagnostic or operative hysteroscopy that required cervical dilation. The efficacy of the device was evaluated by the preforming physician based on three categories:

a) general ease of use,

b) ease of insertion of the catheter along the cervix, and

c) confidence with the procedure. Each category was rated using a numerical score of 1-4: 1=Unacceptable, 2= Difficult, 3=Acceptable, and $4=$ Good.

Results: There were no reported complications in any of the 19 procedures using the Aqueduct 100 dilator. By case 8 and all subsequent procedures, the performing physician then scored a 4 in all categories. Obtaining the desired cervical dilation with this device was achieved in all procedures.

Conclusion: Aqueduct-100 device can be used as an alternative for mechanical dilators with potential to prevent uterine and cervical injuries during cervical dilation.

Keywords: Cervix; Cervical dilation; Balloon catheter

\section{Introduction}

Dilating the cervix is necessary for intrauterine diagnostic or operative procedures such as uterine curettage or performing different procedures such as myomectomy, lysis of adhesion, ablation of endometrium, incision pf septum, etc. In the United States alone three million gynecological procedures are performed annually that will require cervical dilation [reference]. Cervical dilation is generally obtained through either an osmotic method (laminaria) or mechanical method (Hegar, Prat, or Hank dilators), however disadvantages of these techniques include the need for multiple office visits, risks of infection, allergic reactions, cervical lacerations, uterine perforations, bleeding. Studies demonstrate the most common reported complications with osmotic or mechanical methods occur before hysteroscopy, during dilation are uterine perforation and cervical trauma [1-7]. We probably need to talk here how Aqueduct- 100 works and probably decrease the chance for cervical laceration and uterine perforation. (for example, this device needs only one-time insertion, only needs to pass internal OS, gradually dilate the cervix with even pressure, etc). 


\section{Materials and Methods}

\section{Device characteristics}

This study was based on the Aqueduct-100 cervical dilator (please put the name of the company here), a triple-balloon catheter that offers dilation with one insertion and adjusts to fit the needs of all dilation procedures. The dimensions of the two dilating balloons (diameter and length) were selected in order to enable efficient dilation to all cervices ranging between 1 to $7 \mathrm{~cm}$. The outer diameter of the semi-rigid catheter is 9 French $(3 \mathrm{~mm})$, enabling easy insertion and manipulation of the catheter through the cervical canal. The consistency and endurance of the dilating balloons was previously tested and documented to ensure there was no detected risk for breakage $[8,9]$. The reliability of the Aqueduct-100 cervical dilator was confirmed in vitro and ex vivo by Mazza et al [9].

\section{Study Site}

The study was conducted at New York University (NYU) Winthrop University Hospital: Department of Obstetrics and Gynecology, Mineola, New York. Data was collected by one study coordinator in the hospital operating room. An independent data and safety monitoring board monitored the study and reviewed the protocol compliance and outcome data. The protocol was approved by the institutional review board of NYU-Winthrop University Hospital.

\section{Patients}

Nineteen patients were enrolled in the study using the following inclusion criteria: age 18 and older, subjects undergoing any gynecological procedures for benign or malignant conditions that require cervical dilation, and the ability to sign informed consent form.

\section{Surgical Procedure}

All procedures were performed under general endotracheal anesthesia or mild conscious sedation, by the same surgeon (VM). After anesthesia, patients were placed in dorsal lithotomy position, prepped and draped under sterile conditions. The cervix was then visualized using a bivalve speculum and grasped with a single tooth tenaculum. Initially, the cervical diameter was measured by inserting the smallest Hegar dilator. The Aqueduct-100 catheter, with its balloons deflated (Figure 1), was then inserted through the external and internal cervical os into the uterus. The anchoring balloon was inflated through the "Anchor channel (Figure 2) by using a $2.5 \mathrm{ml}$ syringe with $1.5 \mathrm{ml}$ of saline solution in order to fix the catheter in place. Two elongated dilating balloons are then inflated simultaneously at each end of the cervical canal (internal and external os) by performing 3 consecutive injections of $2.5 \mathrm{ml}$ of saline solution through the Dilation channel, for a total volume of $7.5 \mathrm{ml}$ and internal pressure generated in the balloons of 6 bars (Figures 2\&3). The operator can control the inflation rate and time of dilatation of the cervix. The inflation of the dilating balloon takes between 20 to 30 seconds, and the inflated balloons remained in the cervical canal for 3 minutes. In the next stage, saline solution was injected into the cervical canal between the two dilating balloons through the Infusion channel to wash and lubricate the cervix. As a result, the cervix is dilated radially to the desired diameter. For catheter removal, all balloons are deflated, and the catheter removed. Prior to proceeding with the intrauterine procedure, the final diameter of the cervix (internal and external os) was measured by inserting Hegar dilators of increasing size until resistance was met.

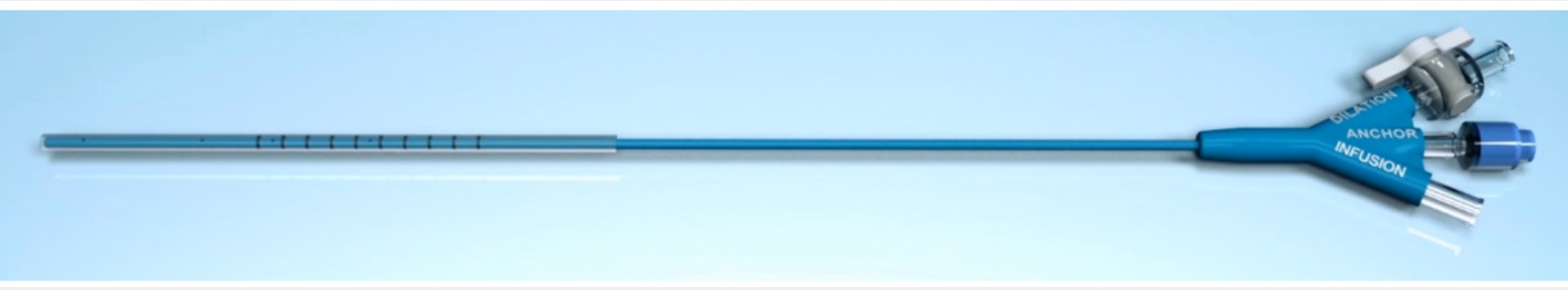

Figure 1: The triple balloon catheter - deflated balloons.

\section{Results}

Nineteen patients were recruited to participate in the study. Their median age was 44 (22-69); 10 patients had history of pregnancy.

\section{Outcome of dilatation}

The averageinitial diameter of the internal os was $1.47 \pm 0.51 \mathrm{~mm}$ and the final diameter was $7.52 \pm 1.34 \mathrm{~mm}$. In all patients, the final diameter of the cervix after dilation met the necessary diameter required to perform the diagnostic/operative procedure without the need for further dilation by another method.

\section{Efficacy of the device}

After the procedure, the efficacy of the device was evaluated by performing physician based on three categories: a) general ease of use, b) ease of insertion of the catheter along the cervix, and c) confidence with the procedure. Each category was rated using a numerical score of 1-4: 1=Unacceptable (unable to insert the device), 2= Difficult (device was inserted, however 
was anatomically or technically challenging), 3=Acceptable (no difficulties with insertion, however did not recall all steps with device use), and 4=Good (no forgotten steps with device use and no difficulties with insertion). Similar to any new device, there is a learning curve for utilizing Aqueduct-100 dilator. In our study, the physician needs to perform as least 8 cases before scoring 4-Good for each category ( Table 1, Again, I suggest we make a table or graph to list the score for every category for every patient, since we do not have any other solid data. ) There were no failed attempts to achieve cervical dilation and no intra- or post- operative complications, including hemorrhage, cervical lacerations or uterine perforations.

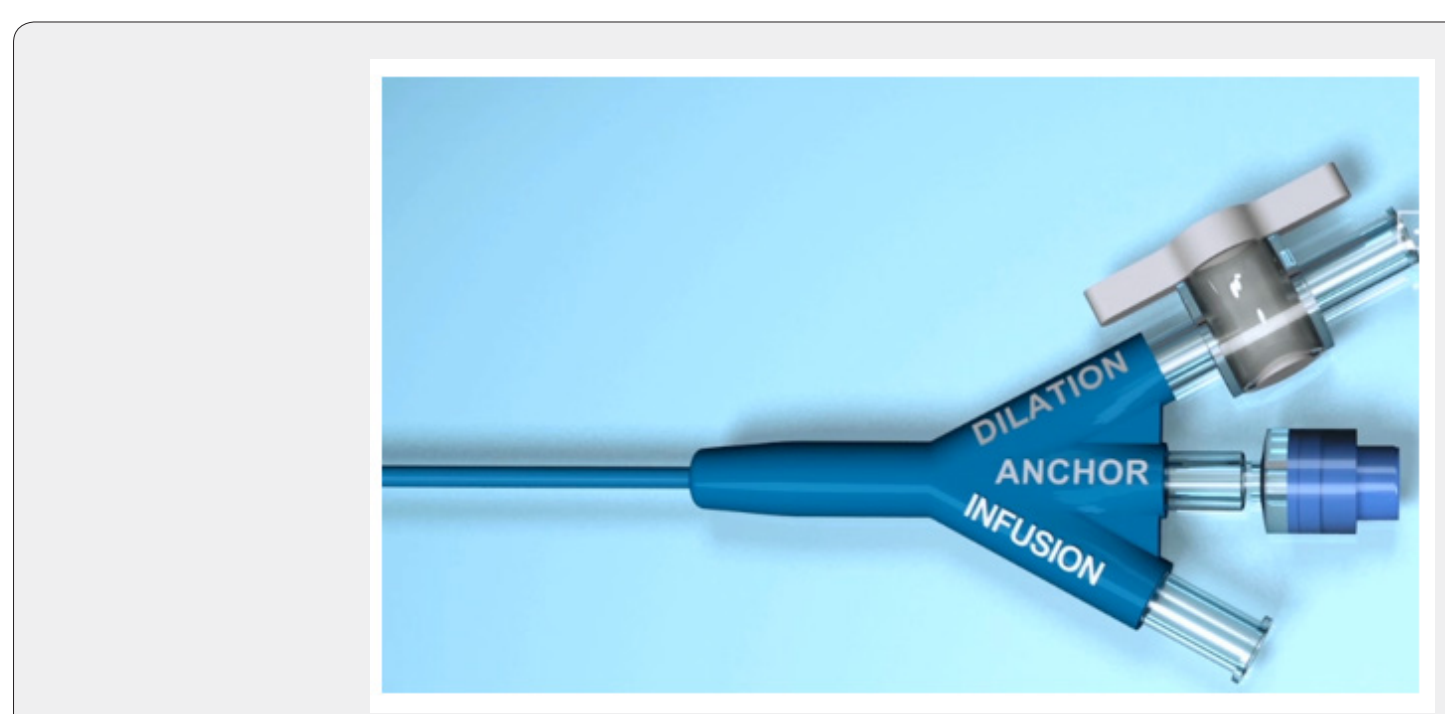

Figure 2: The tree injection channels of the catheter.

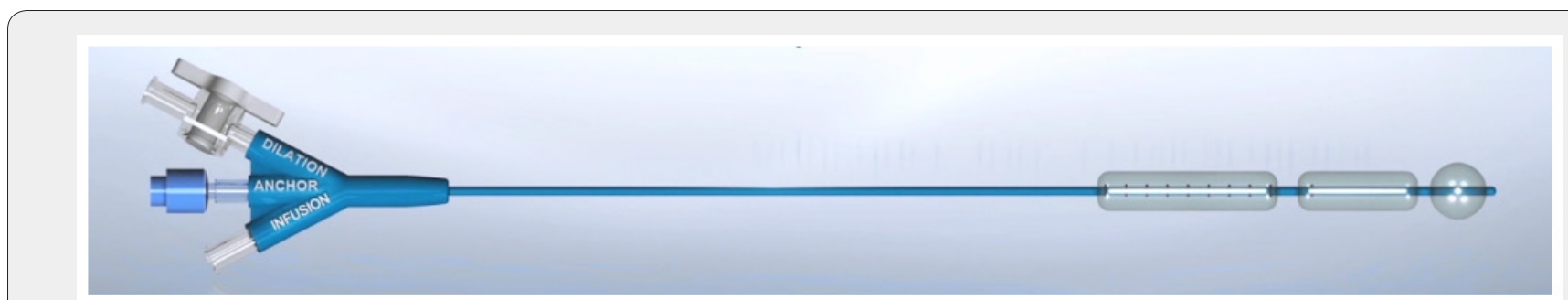

Figure 3: The triple-balloon catheter - inflated balloons (frontal anchor balloons and two elongated dilating balloons).

Table1:

\begin{tabular}{|c|c|c|c|}
\hline & Number of Patients & Mean Diameter (mm) & Standard Deviation \\
\hline Initial Measurement & 19 & 1.47 & 0.51 \\
\hline Final Measurement & 19 & 7.52 & 1.34 \\
\hline
\end{tabular}

\section{Discussion}

The most common methods of cervical dilation are the use of osmotic or sequential mechanical dilators. The major disadvantage of these two devices are that, due to the required physical force needed to insert these dilators, there exists a risk of cervical lacerations, creation of a false passage, and uterine perforations

Two previous studies highlight the importance of cervical resistance in relation to mechanical dilation [10, 11,12]. The minimum pressure needed to overcome cervical resistance was determined using a continuous controllable balloon dilator for 23 seconds under a pressure of 3.8 bars as shown in Arsenijevic et al. While using Aqueduct-100, the two dilating balloons generate a pressure of 6 bars during the 10 seconds of dilation. Therefore, Aqueduct-100 can generate enough pressure to overcome the cervical resistance in a very short of time. Since this device just passes the internal OS and does not need multiple pass of the cervix, the possibility of uterine perforation is very minimal. In addition, the chance of cervical laceration is significant less by dilating the balloon in slow motion. All those benefits were 
demonstrated in Siegelman's study. Provides successful dilation with one insertion, is a device that is user friendly, and requires little physical force even in patients with cervical stenosis [8].

Another advantage of Aqueduct-100 is that the final cervical diameter can be easily controlled by dilation time. A previous study with the Aqueduct-100 dilator was conducted to assess cervical dilation with increasing time ( 3 minutes vs 5 minutes). Fifty patients were divided into 2 groups. Group A ( $n=38)$ underwent cervical dilation with dilating balloons remaining inflated inside the cervix for five minutes and Group B ( $n=12)$ underwent cervical dilation with dilating balloons remaining inflated inside the cervix for 3 minutes. The final diameter was $6.4 \pm 0.8 \mathrm{~mm}$ Group A $(\mathrm{P}<0.0001)$ and $8.1 \pm 1.1 \mathrm{~mm}$ Group B [11]. Interestingly we showed a learning curve of Aqueduct-100 in our study. Although there were no reported problems using the device, we attribute lower scores during the first 8 cases with a learning curve for operator to use the device. Provider confidence of the procedure was scored as 3 acceptable meaning that the operator understood procedural steps however needed the practice of multiple uses to be proficient. After these first 8 cases, the operator scored the use of the device as 4 in all categories. The limitation of our study is the small number of participants, however our results are still very convince that Aqueduct-100 is a simple procedure with high efficacy and possible less complications.

\section{References}

1. Gupta JK, Johnson N (1992) Should we use prostaglandins, tents or progesterone antagonists for cervical ripening before first trimester abortion? Contraception 46: 489-497.
2. Biron-Shental T, Fishman A, Fejgin MD (2004) Medical and mechanical methods for cervical ripening. Int J Gynaecol Obstet 85: 159-160.

3. Lichtenberg ES, Paul M, Jones H (2001) First trimester surgical abortion practices: a survey of national abortion federation members. Contraception 64(6): 345-352.

4. Sutkin G, Capelle SD, Schlievert PM, Creinin MD (2001) Toxic shock syndrome after laminaria insertion. Obstet Gynecol 98(5 Pt 2): 959961.

5. Nguyen MT, Hoffman DR (1995) Anaphylaxis to laminaria. J Allergy Clin Immunol 95(1 Pt 1): 138-139.

6. Gelber S, Sciscione A (2006) Mechanical methods of cervical ripening and labor induction. Clin Obstet Gynecol 49(3): 642-657.

7. Hulka JF, Lefler HT Jr, Anglone A, Lachenbruch PA (1974) A new electronic force monitor to measure factors influencing cervical dilation for vacuum curettage. Am J Obstet Gynecol 120(2): 166-173.

8. Spiegelman L, Sanchez-Rauder, R and Travieso C (2008) Aqueduct-100, A New Device and Method for Controllable Cervical Dilation. J Clin Res Med 1(3): 1-4.

9. Mazza E, Nava A, Bauer M, Winter R, Bajka M, et al. (2006) Mechanical properties of the human uterine cervix: an in vivo study. Med Image Anal 10 (2): 125-136.

10. Arsenijevic S, Vukcevic-Globarevic G, Volarevic V, Macuzic I, Todorovic P, et al. (2012) Continuous controllable balloon dilation: a novel approach for cervix dilation Trials 13: 196.

11. Vico de Miguel FJ, Pantoja-Garrido M, Frias-Sanchez Z, Pantoja-Rosso FJ, Martins E (2017) Experience with a device for controlled cervical dilation. Ginecol Obstet Mex 85(3): 125-133.

12. Tzabari A, Weichselbaum A, Stark M (2018) Towards safe and efficient cervical dilatation. Mini-invasive Surg 2.

\section{Your next submission with Juniper Publishers} will reach you the below assets

- Quality Editorial service

- Swift Peer Review

- Reprints availability

- E-prints Service

- Manuscript Podcast for convenient understanding

- Global attainment for your research

- Manuscript accessibility in different formats

( Pdf, E-pub, Full Tsext, Audio)

- Unceasing customer service

Track the below URL for one-step submission https://juniperpublishers.com/online-submission.php 